\title{
Paragastric, lesser omentum neural block to prevent early visceral pain after laparoscopic sleeve gastrectomy: A randomized clinical trial protocol
}

\author{
Bloqueo neural del epiplón menor paragástrico para prevenir el dolor \\ visceral temprano después de una gastrectomía en manga laparoscópica: \\ Protocolo de un ensayo clínico aleatorizado
}

\author{
Jorge Daes ${ }^{1} \mathbb{D}$, Rafael Pantoja ${ }^{2}$, Andrés Hanssen$^{1}$, Elika Luque$^{1}$, David Morrell $^{3} \mathbb{D}$, \\ Eric M Pauli ${ }^{3}$ iD
}

1 MD, Division of Minimally Invasive and Bariatric Surgery, Department of Surgery, Clínicas Portoazul e Iberoamérica, Barranquilla, Colombia.

2 MD, Department of Anesthesia, Clínicas Portoazul e Iberoamérica, Barranquilla Colombia.

3 MD, Division of Minimally Invasive and Bariatric Surgery, Department of Surgery, Penn State Health Milton S. Hershey Medical Center, Hershey, PA, USA.

\section{Introduction}

The somatic pain induced by surgical trauma to the abdominal wall after laparoscopic sleeve gastrectomy (LSG) is effectively managed using conventional analgesia and transversus abdominis plane (TAP) blocks. In contrast, the visceral, colicky, pain that patients experience after LSG does not respond well to traditional pain management. Patients typically experience epigastric and retrosternal pain that begin immediately after LSG and lasts up to 72 hours after LSG. This visceral type of pain has been ascribed to the spasm of the neo-gastric sleeve. The pain is often severe and requires opioid derivatives. Patients frequently have associated autonomic symptoms such as nausea, retching and vomiting. In the last 15 years at our institutions, we have used many analgesic strategies to manage this burdensome symptom in the more than 2000 LSG procedures we have performed, but none have been satisfactorily effective ${ }^{1,2}$.

Pathways for visceral sensation are diffusely organized both peripherally and centrally. Although the stomach possesses intrinsic neural plexuses that allow some degree of autonomy, it largely depends on extrinsic neural inputs,

Keywords: bariatric surgery; gastrectomy; postoperative pain; visceral pain; pain management; nerve block.

Palabras clave: cirugía bariátrica; gastrectomía; dolor postoperatorio; dolor visceral; manejo del dolor; bloqueo nervioso.

Received: 08/08/2021 - Accepted: 08/18/2021 - Date of publication online: 09/01/2021

Corresponding author: Jorge Daes, Carrera 50 \# 79 - 223 PH B, Barranquilla, Colombia. Phone number: +57 3106363636, Fax: +57 53604666. Email: jorgedaez@gmail.com

Cite as: Daes J, Pantoja R, Hanssen A, Luque E, Morrell D, Pauli EM. Paragastric, lesser omentum neural block to prevent early visceral pain after laparoscopic sleeve gastrectomy: A randomized clinical trial protocol. Rev Colomb Cir. 2022;37:27-32. https://doi.org/10.30944/20117582.1017

This is an open access article under Creative Commons License - BY-NC-ND https://creativecommons.org/licenses/by-ncnd/4.0/deed.es 
particularly those of the parasympathetic and sympathetic pathways. With its afferent pathways stationed at the celiac ganglion, the sympathetic nervous system exerts a predominantly inhibitory influence over gastric musculature and motility. In contrast, the parasympathetic nervous system, consisting of the vagus nerves and its branches, exerts a predominantly excitatory influence over gastric tone and motility ${ }^{3}$. The anterior and posterior vagus nerves run alongside the lesser curvature of the stomach and branch distally. Sympathetic nerves usually accompany the blood vessels. The proposed main mechanisms of action of a paragastric block are a reduction in the parasympathetic influence over the stomach, thus reversing its increased muscular tone and deactivating mechanosensitive receptors in the organ wall, and blockade of the afferent sympathetic fibers that convey visceral pain to the spinal cord ${ }^{4}$.

To address this burdensome symptom, we performed paragastric, lesser omentum neural blocks in a pilot, observational study involving 35 patients. This was done through blockade of the paragastric lesser omentum with $20 \mathrm{cc}$ of $0.5 \%$ bupivacaine, a long-acting local anesthetic, in addition to the routinely performed TAP blocks. We observed a remarkable improvement in the visceral pain level referred to the epigastric and retrosternal areas and associated autonomic and emotional symptoms. The effect was more noticeable during the first few hours after surgery but persisted throughout the early postoperative period. The need for analgesics and the presence of nausea and vomiting were also significantly reduced. These effects were observed even after reducing the anesthesia-administered analgesia protocol. Additionally, a 10-mmHg reduction in the median blood pressure and a 10 beats/minute reduction in the median heart rate 5 to 10 minutes after the blockade were observed.

\section{Objective}

The purpose of this study is to evaluate the safety and effectiveness of the paragastric lesser omentum nerve block in the management of visceral pain in the early postoperative period after LSG. We hypothesize that the proposed nerve block is safe, reduces visceral pain and associated autonomic symptoms, and provides hemodynamic stability in the early postoperative period after LSG.

\section{Methods}

\section{Study design}

The herein-described study is a prospective, doble-blinded, randomized clinical trial involving patients undergoing LSG at two institutions: Clínica Portoazul and Clínica Iberoamérica in Barranquilla, Colombia. The patients will be randomized to one of two parallel groups: LSG with laparoscopic TAP block only and LSG with laparoscopic TAP block and an additional paragastric lesser omentum neural blockade. The study protocol will adhere to the Helsinki Declaration and will be reviewed by the Clínicas Portoazul and Iberoamérica Institutional Review Board and Ethics Committee. The study will comply with the CONSORT 2010 guidelines for the reporting of randomized clinical trials. A flow diagram of progress through the phases of the parallel randomized trial of two groups is found in figure 1. Two assistants will schedule eligible patients according to hospital availability and patient convenience.

\section{Patients}

All patients scheduled for LSG at one of the participating institutions and that consent to study participation will be eligible for screening for study inclusion. The exclusion criteria will be need for revisional surgery, need for concomitant hiatal hernia repair or other surgical procedures, conversion to open surgical procedures, allergies to nonsteroidal anti-inflammatory drugs or acetaminophen, intraoperative or postoperative complications (e.g., visceral or vascular perforations, large hematomas, or hemorrhage requiring transfusion or reoperation), sepsis, pulmonary embolism or anesthesia-related complications requiring intensive care admission.

\section{Study Outcome}

The primary outcome will be patient-reported pain scores using an 11-point visual analog pain scale. The secondary outcomes will be analgesic 


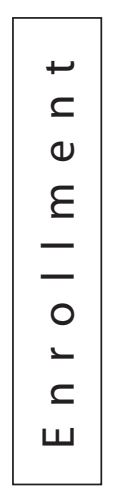

Assessed for eligibility $(n=)$
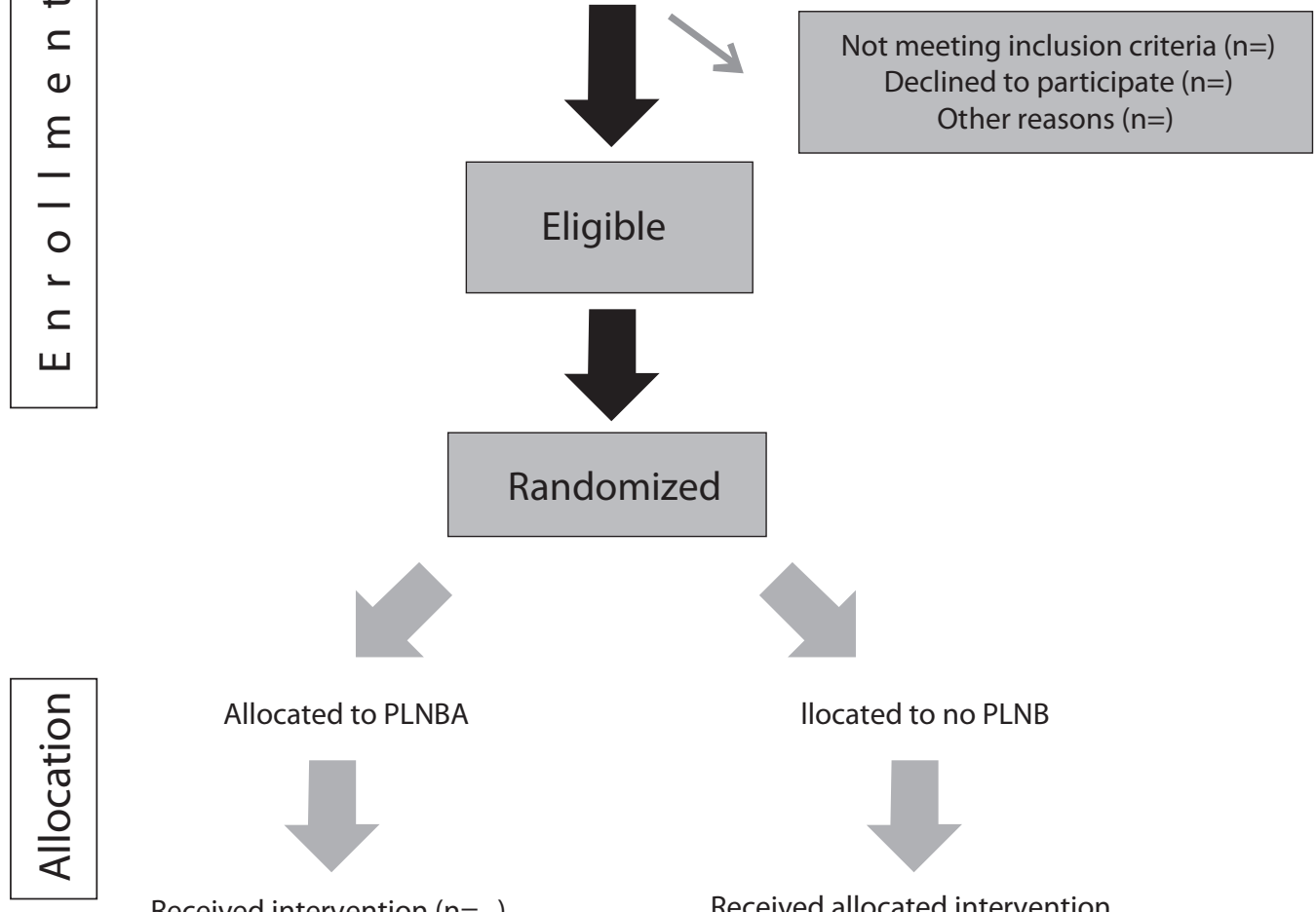

Allocated to PLNBA

llocated to no PLNB

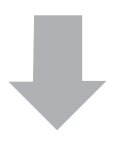

Received intervention $(\mathrm{n}=$...)

Did not (reasons) $(\mathrm{n}=\ldots)$

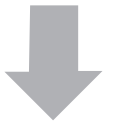

Lost of follow up (reasons) $(n=\ldots)$

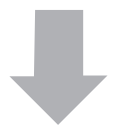

Analyzed $(\mathrm{n}=\ldots)$

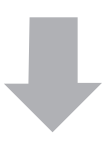

Received allocated intervention

Did not (reasons) $(\mathrm{n}=\ldots)$

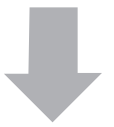

Lost of follow up (reasons) $(n=\ldots)$

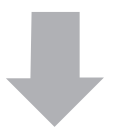

Analyzed $(\mathrm{n}=\ldots)$

Excluded from analysis (reasons) $(n=\ldots) \quad$ Excluded from analysis (reasons) $(n=\ldots)$

Figure 1. Flow diagram of the progress through the phases of a parallel randomized trial of two groups (i.e., enrollment, intervention allocation, follow-up, and data analysis). PLNB, paragastric lesser omentum nerve block.

requirements; the presence of nausea, vomiting, hiccups, tachycardia, hypertension, or urinary retention; oral tolerance; and the changes in the mean arterial blood pressure and median heart rate 5 minutes after blockade. Outcomes will be assessed during the period of inpatient admission following LSG.

\section{Sample Size}

Previously published data have indicated that differences of 1 to 2 on an 11-point visual analog pain scale are clinically significant ${ }^{5-7}$. Based on these prior studies, we chose a difference of 1 as the minimum clinically significant difference for purposes of sample size calculation and assumed a 
standard deviation of 2 . With a p-value of 0.05 and a power of 0.80 , we estimated that a total of 128 patients would need to be enrolled in this study. To allow for any potential loss to follow-up, we plan to enroll 150 patients in the study.

\section{Randomization and Blinding}

Randomization will be performed using sealed envelopes, stratified by institution in blocks of six. These envelopes will be prepared by the data manager. The randomization list will be stored by the data manager (PF) containing final treatment assignments. Only the data manager will have access to the randomization list throughout the study. These sealed envelopes will be placed in the patients chart and will not be opened until the patient is in the operating room and general anesthesia has been induced. Both the patient and the investigator assessing the patient postoperatively will be blinded to treatment arm assignments.

\section{Data Collection}

Patient age, sex, body mass index, current medications, and medical and surgical history will be recorded prospectively at the preoperative clinic visit at the time of study enrollment with informed consent. An analog pain scale survey will be administered by an investigator blinded to the patient's group at 1 hour in the recovery room, at 8 hours after surgery, and at the following morning. The investigator will record the need for analgesics; oral tolerance; the presence of nausea, vomiting, retching, excessive salivation and hiccups; vital signs; and the presence of spontaneous diuresis.

\section{Statistical analysis}

Continuous outcome variables will be compared with two-sample t-tests. Categorical and binary outcome variables will be compared using chi-squared tests. Patient-reported pain scores will be further compared using linear regression with a history of chronic pain, use of narcotics, and the surgeon who performed the operation as covariates.

\section{Surgical procedures LSG technique}

The LSG technique performed by our group has previously been described elsewhere ${ }^{1,2}$. Three surgeons from our group (JD, EL and AH) with extensive experience in LSG will perform all procedures. Briefly, we use five ports as follows: a 12 -mm port at the umbilicus for the laparoscope insertion and for stapling and removal of the stomach; a second 12-mm port at the left flank for devascularization of the greater curvature, for suturing and as a secondary position for insertion of the laparoscope during stapling; and three additional 5-mm ports, one at the epigastrium for liver retraction, one at the right upper quadrant for the surgeon's left-hand working trocar, and one at the left lateral subcostal area for the assistant.

The greater curvature is devascularized using an ultrasonic device starting $3 \mathrm{~cm}$ proximal to the pylorus and continuing until the fundus is dissected free of the left crus of the diaphragm. We ensure an intra-abdominal esophagus. When a hiatal hernia is present, we completely free the esophageal-gastric union from the left and right crura, divide the phrenoesophageal membrane and periesophageal connective tissues, and continue the dissection well into the mediastinum to ensure a sufficient length of intra-abdominal esophagus. The hiatal hernia defect is then closed with nonabsorbable monofilament sutures. Neither a calibrating bougie nor mesh is used, even for large hernias for which a Hill procedure is added.

A 36-French bougie is introduced through the esophagus and into the duodenum. Division of the stomach starts $1 \mathrm{~cm}$ proximal to the pylorus, keeping the bougie adjacent to the lesser curvature. We fire four to five staple cartridges $(60 \mathrm{~mm}$ length, $3.5-4.8 \mathrm{~mm}$ height), carefully avoiding relative narrowing at the junction between the vertical and horizontal parts of the stomach, which usually occurs during the first, and occasionally the second, firing of the stapler if it is inappropriately pressed against the bougie in an attempt to leave a small antrum. Narrowing can be avoided by using an articulating stapler that is slightly angled to the 
greater curvature, to create a wide angle at the junction between the horizontal antrum and the vertical body of the stomach.

When the pylorus is located in the right upper quadrant and the stomach is very curved, the only way to form a small antrum and avoid relative narrowing is to place an additional trocar through a port at the right flank to help aim the first stapler correctly.

When firing the staplers, it is important not to overstretch the stomach to avoid narrowing by the recoil of the stapler line. The anterior stomach wall and posterior stomach wall must be equal and flat to keep the sleeve from rolling or spiraling, which will result in food intolerance or gastroesophageal reflux disease. Division of the stomach, including most of the fundus, is then completed, leaving only a small portion for oversewing.

We bury the staple line with continuous seromuscular stitches, using nonabsorbable monofilament sutures, starting at the top of the sleeve. We then retract the bougie proximally, test the sleeve with 25 to $35 \mathrm{~mL}$ of methylene blue (more to ensure proper distal flow of the fluid), and, after incising the aponeurosis, extract the stomach through the umbilical port without an endoscopic retrieval bag. Trocars are removed under direct vision to ensure hemostasis and the aponeurosis is sutured.

\section{Laparoscopic TAP block}

The laparoscopic TAP block is performed after testing the sleeve with methylene blue. We infiltrate $40 \mathrm{~mL}$ of $50 \%$ diluted $0.5 \%$ bupivacaine in the posterolateral subcostal area on both sides. We confirm infiltration into the proper plane by observing the correct dissemination of the fluid between the muscle layers.

\section{Paragastric, lesser omentum neural block}

The paragastric, lesser omentum neural block is performed with a 25-gauge needle attached to a venous catheter extension introduced through the left $12-\mathrm{mm}$ port. The needle is capped during its introduction and the cap is removed inside the abdomen using a grasper and kept under direct vision at all times. Infiltration of $20 \mathrm{~mL}$ of non-diluted $0.5 \%$ bupivacaine is performed at five levels in the fatty tissue of the paragastric area with careful aspiration preceding the infiltration of fluid. We ensure proper infiltration of the lesser omentum at its insertion to the stomach wall at the esophagogastric union, mid-stomach, and distal antrum. Next, we infiltrate the hepatoduodenal ligament. Finally, the posteriorsuperior paragastric area is infiltrated (this area is accessed by elevating the proximal half of the sleeve from the stomach's neo greater curvature). The cap is then reapplied to the needle, and the assembly is removed from the abdominal cavity. A video of the procedure is available at https://youtu.be/Rx08blhLLAs.

\section{Recovery Protocol}

All patients will receive proton pump inhibitors, conventional antiemetics, and a baseline analgesic such as acetaminophen $1 \mathrm{gr}$ intravenously every 6 hours, or dipyrone 1 gr intravenously every 6 hours. The first-line rescue analgesics will be a nonsteroidal anti-inflammatory drug such as diclofenac (given intravenously every 12 hours) together with hyoscine butyl bromide 20 mgs (given intravenously every 12 hours), and the second line rescue analgesic will be tramadol (1 mg per ideal weight intravenously every 6 hours). A water popsicle will be offered the afternoon after surgery, and clear fluids will be started the following day after surgery. Patients will be discharged from the hospital at noon the day after surgery.

\section{Discussion}

Reducing severe visceral pain and its related side adverse effects that are characteristically seen in the early postoperative course after LSG may impact recovery room time, medication costs and side effects, nursing time allocation, narcotic abuse, and most importantly, the patient's wellbeing and comfort. This study will lead to related investigations such as:

1. The exploration of strategies to extend the duration of the blockade (e.g., use of liposomal bupivacaine, colloids and adrenaline, or 
creation of blisters of local anesthetics in the area);

2. The performance of an early paragastric block (i.e., before the staple division of the stomach) to determine whether the often-seen hemodynamic changes precipitated by the stomach division are obliterated (such as tachycardia and hypertension);

3. Measurement of the manometric changes that occur in the stomach after paragastric neural block;

4. Testing of the use of atropine to replace the neural block if this proves effective;

5. Using a similar block to address visceral pain after other gastrointestinal (colon cancer, pancreatic resections) and genitourinary procedures, opening a new chapter in the management of visceral pain after abdominal procedures.

\section{Conclusion}

Preliminary results suggest that the paragastric neural blockade is safe and may be effective in addressing the visceral pain and associated autonomic changes that accompany the early postoperative course of patients after LSG. Here we propose a randomized clinical trial to evaluate the value of this new strategy.

\section{Compliance with ethical standards}

All medical and surgical procedures will be performed in accordance with the ethical standards of the institutional research committee for studies involving human participants and with the 1964 Helsinki declaration.

Informed consent: Informed consent will be obtained from all individual participants included in the study.

Conflict of interest: The authors of this manuscript have no conflict of interest in relation to this publication.
Funding sources: The study received no external funding.

\section{Authors' contributions:}

Conception of the study: Jorge Daes

Design of the study: Rafael Pantoja, Andres Hanssen, Elika Luque

Data acquisition: N/A

Data analysis and interpretation: David Morrell

Drafting of the manuscript: Jorge Daes, Andres Hanssen

Critical review: Eric M. Pauli

\section{References}

1. Daes J, Jimenez ME, Said N, Daza JC, Dennis R. Laparoscopic sleeve gastrectomy: symptoms of gastroesophageal reflux can be reduced by changes in surgical technique. Obes Surg. 2012;22:1874-9. https://doi.org/10.1007/s11695-012-0746-5

2. Daes J, Jimenez ME, Said N, Dennis R. Improvement of gastroesophageal reflux symptoms after standardized laparoscopic sleeve gastrectomy. Obes Surg. 2014;24:536-40. https://doi.org/10.1007/s11695-013-1117-6

3. Browning KN, Travagli RA. Central nervous system control of gastrointestinal motility and secretion and modulation of gastrointestinal functions. Compr Physiol. 2014;4:1339-68. https://doi.org/10.1002/cphy.c130055

4. La J-H, Gebhart GF. Visceral pain. In: Aminoff MJ, Daroff RB, editors. Encyclopedia of the neurological sciences. 2nd ed. Cambridge, MA: Academic Press; 2014. p. 672-6.

5. Kelly AM. Does the clinically significant difference in visual analog pain scores vary with gender, age, or cause of pain? Acad Emerg Med. 1998;5:1086-90. https://doi.org/10.1111/j.1553-2712.1998.tb02667.x

6. Kelly AM. The minimum clinically significant difference in visual analog scale pain score does not differ with severity of pain. Emerg Med J. 2001;18:205-7. https://doi.org/10.1136/emj.18.3.205

7. Gallagher EJ, Bijur PE, Latimer C, Silver W. Reliability and validity of a visual analog scale for acute abdominal pain in the ED. Am J Emerg Med. 2002;20:287-90. https://doi.org/10.1053/ajem.2002.33778 